\title{
Present Situation and Transformation Development of High Polymer Materials Industry in China
}

\author{
Hongdan Hu, Yaowen Wang
}

Jiangsu College of Safety Technology, Xuzhou City, Jiangsu Province, 221011, China

\section{Keywords: Polymer materials; Industry status; Green development; Transformation}

\begin{abstract}
As one of the friendly materials, polymer material has become a hot research topic at home and abroad. Based on the author's learning and practical experience, this paper firstly analyzed production status and existing problems of three kinds of synthetic materials in China, and then discussed the research status of polymer materials; finally, we have proposed the direction of technological innovation of polymer materials. Therefore, in this paper, we believed that there is enough space for innovation in the field of polymer materials, which can pass the development of new products of high performance "green" polymer materials and "green" polymer so that to realize the transition of polymer material industry into innovation driven development in China.
\end{abstract}

\section{Introduction}

After experiencing the three stages of technology introduction, technology localization and technological innovation, China's polymer materials industry is now in a critical period of transformation and development. In the early stage of development, China's various types of polymer materials markets are in short of supply, and the product profit space is very large and the production of general products can create huge profits. However, with the low cost of natural gas in the Middle East, the United States shale gas, the rapid development of the domestic coal chemical industry as a raw material polymer material and substantial increase in natural rubber production capacity, the global production capacity of polymer materials has been a serious surplus trend and the former glorious market environment has ceased to exist.

\section{Production Status and Existing Problems of the Top Three Synthetic Materials in China}

Since 2009, China has become the world's largest producer and consumption countries of synthetic rubber. In 2013, China's total consumption of synthetic rubber is 4270kt. Among them, there are $26 \%$ of imported rubber and the consumption of natural rubber attached to $3300 \mathrm{kt}$ (natural rubber imports accounted for $75 \%$ ). The production of synthetic rubber is currently in a state of serious loss, which is difficult to improve its profitability in a very short term.

Since 2010, China has become the world's largest producer and consumer of synthetic resins. In 2013, the apparent consumption of synthetic resin reached 76650kt; among them, 23.9\% of synthetic resin from imports. At present, polyvinyl chloride, polyvinyl alcohol and polyvinyl acetal resin prices have fallen sharply, which caused a serious loss. Polyethylene and polypropylene resin production capacity increased rapidly, there is a great possibility to repeatative formation of synthetic fiber and synthetic rubber as tragedy momentum. People became optimistic about the polypropylene resin production capacity in the past, which now has exceeded consumption since 2012. But the high-end products must also depend on import. Due to the rapid development and production capacity exceeds demand, polypropylene production has reached the edge of loss. But China's new polyethylene and polypropylene production capacities are still being released; the polypropylene resin production capacity 
increased by $3080 \mathrm{kt}$ in the second half of 2014 and the polyethylene resin production capacity increased by $1500 \mathrm{kt}$.

It is obvious that excess capacity is the main problem of polymer material industry in China. On the one hand, the high cost of low-end products with low competitiveness, and the production capacity has been surplus; on the other hand, due to the lack of high-end products and slow development, it need to rely on imports, which further exacerbate overcapacity. It is not difficult to see that the outlet for transformation development of China's polymer materials industry is to solve the problem of excess capacity fundamentally. The problem of excess capacity can be solved from two aspects: first, expand demand through exports; second, develop the high-end products through technological innovation so that to reduce imports. Both complement each other, and are indispensable.

China's existing polymer material industry cost advantage is not obvious. Therefore, in order to make the products can be exported on a large scale, it is necessary to develop high-end products with high performance, high added value and comparative advantage. These high-end new products must occupy the global market to form economic scale. Thus, the high-end products and the internationalization of the market will become China's important and even be the only direction of development. However, if we want to achieve the transformation of the product, we must firstly analyze the country whether has such a basis and ability to innovate, and whether the market has enough space for technological innovation. Fortunately, with more than thirty-year of reform and opening up, China has established a good technical basis in the study of polymer materials, and the development of the three synthetic materials still has enough space for technological innovation. As long as change the concept and rationalize mechanism, it is entirely possible that China's three major synthetic materials can usher in new opportunities for development and create new glories.

\section{Research Status of High Polymer Materials}

Existing technologies. In polymer physics, domestic researchers have carried out a systematic study on the relevant polymer physics and its applications through a series of national key basic research and development projects, which provides a solid theoretical basis for the development of high performance of the polymer materials. For example, the project of "basic research on high performance of polymer materials", which not only applies the basic theory research achievements to the development of high performance biaxially oriented polypropylene (BOPP) resin and high performance PE-100 pipe resin, but also equip polymer material production enterprises with the ability of drawing inferences.

In the aspect of polyolefin catalyst, the research level of China has been in the international advanced level. As a result of the introduction of the almost all of the polyolefin production process in the world by domestic petrochemical enterprises, which has led to the study of catalysts in China involving almost all types of catalysts around the world. Sinopec Beijing Chemical Research Institute has developed a series of new catalyst technology on the platform of $\mathrm{N}$ catalyst production technology and the new technology of glycol ester. The commercially available high performance polypropylene catalysts include $\mathrm{N}$ catalyst, DQ catalyst, ND catalyst, HA catalyst with high activity, BCM catalyst which can be polymerized at high temperature and so on; High performance polyethylene catalysts include BCE catalyst, BCL catalyst and CMU catalyst for the production of ultra-high molecular weight polyethylene. The catalyst is not only applied to the domestic polyolefin production equipment, but also exported to the United States and other developed countries.

In polyolefin polymerization process, China initially only relys on the introduction of technology. In recent years, China gradually realized the localization of the main technology and has successfully developed a number of original technology through the unremitting efforts 
of scientific and technological personnel. For example, a new process for the asymmetric addition of polypropylene to the electron, a new process of high temperature polymerization of polypropylene, a new process for the third generation of tubular polypropylene and a new process of gas liquid solid three phase ethylene polymerization. These new technologies have been used to develop a variety of products with high performance and low cost.

Technological innovation space. In recent years, China has developed many popular new products in the domestic market, such as high speed BOPP homopolymer resin, direct polymerization of high melt strength polypropylene resin, low volatile organic compounds (VOC) of high impact polypropylene resin, transparent high impact polypropylene resin, phthalate free, two carbamate (Plasticizer) polypropylene resin, low soluble content propylene / butylene random copolymer (G- resin), high speed biaxially oriented polyethylene (BOPE) film special resin, special resin for polypropylene non-woven fabric with narrow molecular weight distribution with no plasticizer, no peroxide (including homopolymer, soft, antibacterial, etc.); in addition, there are antibacterial and antifungal polypropylene resin, high performance aramid fiber, high performance ultra-high molecular weight polyethylene fiber and direct polymerization of high purity polypropylene resin. Among them, the catalyst used in the production of non plasticizer polypropylene resin does not contain the traditional inner electron donor of butyl phthalate two; instead, the use of glycol esters as a catalyst for the removal of sensitive plasticizers can be eliminated. Compared with transparent polypropylene produced by traditional technology, the solubility of G- resin was obviously reduced. Therefore, the application of G- resin in medical devices and food packaging can greatly improve the safety level.

\section{Technical Innovation Direction}

High performance of existing polymer materials. The existing high performance polymer materials should be the most important part, and also should be a priority direction of development. This kind of performance polymer materials include: polar polyolefin new material, high speed and low cost BOPE film special resin, thermoplastic processing of polyvinyl alcohol plastics, high purity polypropylene resin used for capacitor film, low VOC content, no plasticizer, anti-bacterial and special resin polypropylene non-woven fabric, anti-bacterial plastic and fiber, lower soluble transparent high impact polypropylene copolymer with high melt strength, high impact polypropylene resin, high crystallization of high impact polypropylene resin, energy saving automobile tire tread rubber overcoming the "magic triangle", high barrier butyl rubber composite material, engineering plastics PET resin and engineering plastics nylon resin.

High performance green high polymer materials. In order to cope with the fierce market competition and ensure the healthy and sustainable development of polymer material industry in china, high performance green polymer materials must be developed. Such materials include: bio Based Polyamide and other bio based polymer materials, graphene and other carbon materials composite materials, biodegradable agricultural mulch film material with low cost and high strength, polymer material for high grade lubricating grease, poly (1butene) resin, olefin homopolymer or copolymer elastomer, polymer material for 3D printing, thermal conductivity, conductive polymer new materials, energy saving LED lighting and display light diffusion materials, shale oil and gas using polymer new materials and polymer nanocomposites, etc.

"Green" high-end polymer products. "Green" high end polymer products is the field of technology development which must be extended in the healthy development of polymer materials in China. The products include: lithium ion battery polyolefin membrane, ultrafiltration and reverse osmosis membrane separation membrane materials, processing 
technology and materials and products of polyolefin machine drawing, high performance foaming polyolefin material, building exterior insulation material with low thermal conductivity and low cost, high performance and low-cost antistatic fiber, flame retardant and anti dripping PET fiber, antibacterial PET fiber and super Imitation cotton fiber with antibacterial, mildew resistant, flame retardant and anti dripping functions.

\section{Summary}

If we can change the development model, look at the international market and take the innovation as a necessary product rather than luxury, the polymer materials industry will overcome difficulties and there will be the successful realization of the transformation and development. The transformation can not be achieved without the support of national policy. China should control the expansion of low-end industries and encourage the development of high-end industries; import and export management and policy guidance should be strengthened to control the import of low-end products and waste and the government should encourage the export of high-end products. At the same time, we should take advantage of China's institutional advantages; what's more, we should strengthen the production, learning, research and joint research under the guidance of the government so that to improve the technology level of the related industries and the industrial competitiveness fastly.

\section{References}

[1] Zhang Gengming. Characteristics, Application and Development of the High Polymer Materials [J]. Chemical design communication, 2016,(09):37.

[2] Hu Min, Xiang Wei Wei, Tan Jinghua, et al. Development of High Barrier Polymer Packaging Materials [J]. Guangzhou chemical, 2015,(09):10-12+20.

[3] Cai Rushan, Zhang Hongbin. The Development of High Polymer Materials Aging Test and Standard [J]. Environmental technology,2014,(03):59-63.

[4] Tao Guo, Zeng Qi, Xu Youhui, Chen Ziran. Research Status and Development Trend of Polymer Microspheres [J]. Guangzhou chemical,2013,(14):9-10+32.

[5] Shi Lan, Guo Jinyu, Ba La Hari. Development Status and Prospect of High Polymer Antibacterial Materials [J]. Journal of Inner Mongolia University for the Nationalities (Natural Science Edition),2011,(01):25-28.

[6] Yan Zhiyun, Xie Pengcheng, Ding Yumei, Yang Weimin. The Forming and Development of Medical High Polymer Materials [J]. Rubber and Plastics Technology and Equipment, 2010,(12):25-31. 